\title{
Transplantations-Pflegesymposium des AKTX-Pflege e. V. Rückblick auf die Veranstaltung in Dresden
}

Der AKTX-Pflege e. V. veranstaltete am 23.10.2015 im Kongresszentrum Dresden das 19. Pflegesymposium rund um die Transplantation. Den ca. 130 Pflegekräften wurde ein breites und interessantes Spektrum an Vortragsthemen geboten.

Am 23.10.2015 besuchten rund 130 Pflegekräfte im Kongresszentrum Dresden das 19. Pflegesymposium der AKTX-Pflege e.V. Die 1. Vorsitzende des AKTX-Pflege e.V. Gesa Pamperin, die Pflegedienstleitung am Uniklinikum Dresden Ingrid Schultz sowie der DTG-Tagungspräsident Prof. Christian Hugo, Dresden, begrüßten die Teilnehmer.

\section{Interessante Vorträge}

Danach begann das Dresdner Team um Dr. Mirian Opgenoorth, Oberärztin Nephrologie, und Kerstin Borrmann, Stationsleitung Nephrologie, mit der Vorstellung von 2 Fallbeispielen und den beiden Schwerpunkten „Immunologie und Abstoßungstherapie bei Nierentransplantation“ sowie „Vorbereitung und Nachsorge bei Nierentransplantation - Dresdner Konzept“.

Anne Rebefka MSc ANP, Universitätsklinik Freiburg, Doktorandin Cardiff University (UK), folgte mit „Für dieses schöne Geschenk musst du dann nachher bezahlen: Medikamentenadhärenz nach Nierentransplantation, Sichtweise der Patienten“. In der folgenden Session wurde u. a. die aktuelle Situation der Organspende in Deutschland durch den DSO-Koordinator Region Ost Stephan Dammköhler und die neuen Richtlinien der Transplantation durch den Generalsekretär der Deutschen Transplantationsgesellschaft

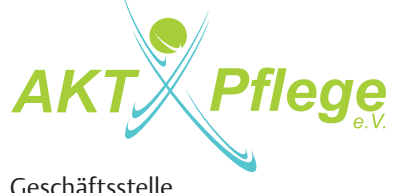

Geschäftsstelle

Arbeitskreis Transplantationspflege e. V. Gesa Pamperin (1. Vorsitzende)

Poppenbütteler Weg 128

22399 Hamburg

E-Mail: g.pamperin@transplantationspflege.de

Internet: www.transplantationspflege.de
Prof. Bernhard Banas, Regensburg, erläutert.

Am Nachmittag war der Themenschwerpunkt die Pädiatrie, in welchem z. B. Tanja Pardela, eine deutsche Transplantationsnurse am Kings College London (UK), ihren Arbeitsalltag in London beschrieb. Weitere Vorträge waren zum Thema Erlebnispädagogik in der Langzeitbetreuung sowie die Vorstellung des Ederhofs, welcher eine familienorientierte Rehabilitation für organtransplantierte Kinder, Jugendliche und junge Erwachsene in Österreich anbietet.

Den Abschluss des sehr erfolgreichen Pflegesymposiums bildeten die thorakalen Organe. Mirko Fahlbusch aus der MHH stellte die Atemtherapie in der Transplantationsmedizin vor. Volker Lauenroth, VAD-Koordinator in Bad Oeynhausen, referierte zum Thema „VAD-Systeme - Einsatzmöglichkeiten und Grenzen“.

\section{Symposium 2016}

Die Planungen für das 20. AKTX-Pflegesymposium haben bereits begonnen. Dieses findet am 06. und 07.10.2016 in Essen statt. Wollen auch Sie Ihre Arbeit, Ihre Erfahrungen und/oder Ihr Zentrum mit einem Poster oder einem Vortrag beim Jubiläumssymposium vorstellen? Reichen Sie den Postertitel oder das Abstract bis zum 10.01.2016 unter info@trans plantationspflege.de ein.

\section{Neues aus dem AKTX-Pflege e. V.}

Im Rahmen des Pflegesymposiums fand auch die diesjährige Mitgliederversammlung statt. Gesa Pamperin stellte u.a. den Tätigkeitsbericht des letzten Jahres vor. Nachdem Britta Nehls als 2. Vorsitzende aus privaten Gründen aus dem Vorstand ausschied, wurde Ulla Busch, Stationsleitung der Nephrologie am Universitätsklinikum Regensburg, als 2. Vorsitzende in den Vorstand gewählt.

Außerdem wurden Kommissionen zu diversen Themen gegründet, so auch z.B. zu Niere, Leber, Reha und Pädiatrie. Aufgabe und Zweck der Kommissionen sind z.B.

- der Austausch untereinander,

- das Verfassen und Veröffentlichen von Artikeln,

- Ansprechpartner für Fragen rund um die Themen sein,

- Referententätigkeiten auf Symposien und

- die Mitgestaltung des Programmes für das jährliche Pflegesymposium.

Die Mitarbeit in den Kommissionen ist auch jederzeit für Nichtmitglieder des AKTX-Pflege e.V. möglich. Weitere Informationen bekommen Sie über info@ transplantationspflege.de oder www. transplantationspflege.de.

Marion Gnädig, Tübingen

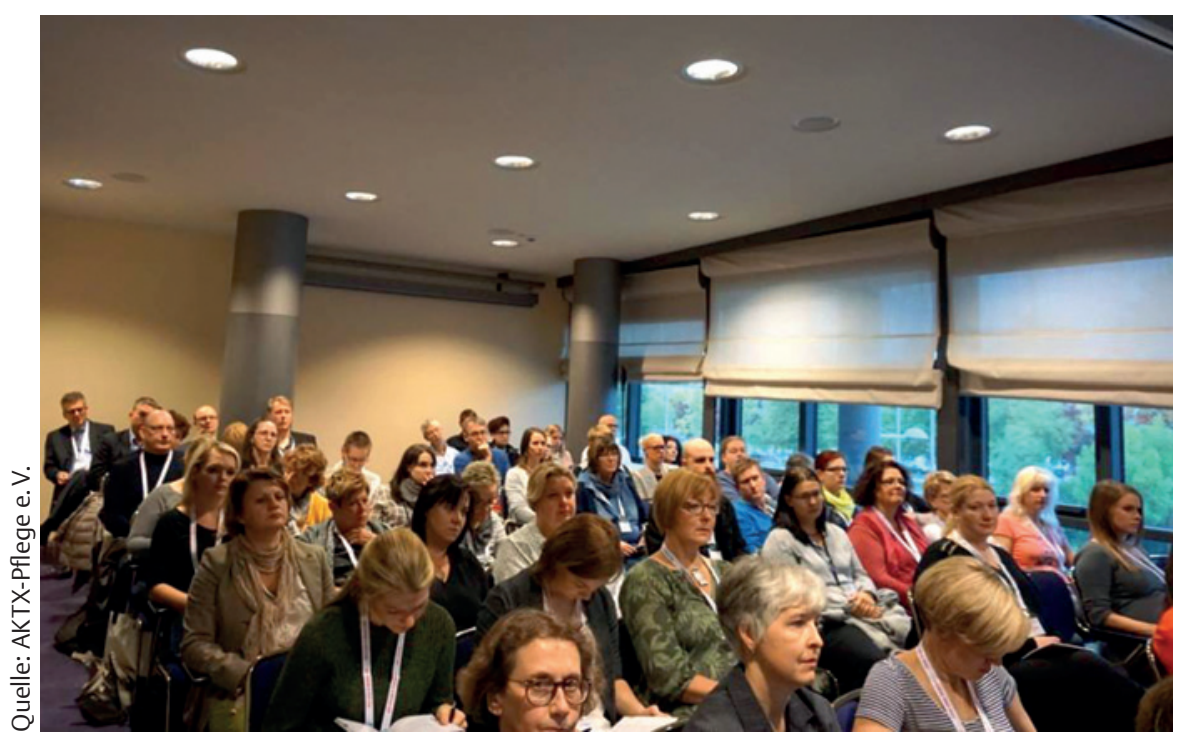

